\title{
Photogrammetric Precise Surveying Based on the Adjusted 3D Control Linear Network Deployed on a Measured Object
}

\author{
Krzysztof Karsznia ${ }^{1 *}$, Edward Osada ${ }^{2}$ \\ 1 Warsaw University of Technology, Faculty of Geodesy and Cartography; Pl. Politechniki 1, 00-661 Warsaw, \\ Poland, krzysztof.karsznia@pw.edu.pl \\ 2 University of Lower Silesia; Faculty of Technical Sciences, Wagonowa St. 9, 53-609 Wrocław, Poland; \\ edward.osada@dsw.edu.pl \\ * Correspondence: krzysztof.karsznia@@pw.edu.pl; Tel.: +48 222347369
}

Featured Application: The elaborated method can be applied to dimension objects, calculate volumes, or measure displacements. The approach uses popular and commonly available instruments making the solution universal.

\begin{abstract}
In surveying engineering tasks, close-range photogrammetry belongs to leading technology considering different aspects like the achievable accuracy, availability of hardware and software, accessibility to measured objects, or the economy. Hence, constant studies on photogrammetric data processing are desirable. Especially in industrial applications, the control points for closerange photogrammetry are usually measured using total stations. In the case of small objects, more precise positions of control points can be obtained by deploying and adjusting a three-dimensional linear network set up on the object. The article analyzes the accuracy of the proposed method, based on the measurement of the linear network using a tape with a precision of $\pm 1 \mathrm{~mm}$. The experiment shows that the adjusted positions of the network control points can be determined with high, onemillimeter accuracy. The photogrammetric 3D model derived referring to such control points and stereo-images captured with a non-metric camera is also characterized by the highest possible precision, which qualifies the presented method to accurate measurements used in surveying engineering. The authors prove that the distance between two randomly optional points derived from the 3D model of a dimensioned object is equal to the actual distance measured directly on it with onemillimeter accuracy.
\end{abstract}

Keywords: Close Range Photogrammetry, 3D linear control network, object dimensioning

\section{Introduction}

Close range photogrammetry (CRP) is a versatile surveying technology applicable to creating object models of different sizes, from small industrial objects to large buildings $[1,2]$. The accuracy of CRP mostly depends on the size of the pixel, the distance between subsequent camera positions, and the respective distances to the photographed object. Moreover, the essential factor is the distribution, density, and accuracy of the control points. These points deployed on engineering objects and captured with CRP and terrestrial laser scanning (TLS) are usually measured using classical methods, such as a total station $[3,4,5,6-7]$. The CRP techniques are used in various engineering tasks related to studying the geometry of objects [8] - both in the macro and micro scale [9, 10]. According to the current state-of-the-art in land surveying and the capacities offered by modern computers, it is possible to utilize CRP while capturing static and dynamic objects being in motion. For example, in publication [11], the authors focused on the self-developed photogrammetric system consisting of dedicated hardware and employing a unique adjustment strategy to determine the accurate position of the mapped objects. They proved that such an integrated approach significantly increases the positioning accuracy allowing for 
fast registration of the existing scenes. The GNSS satellite receiver and the IMU inertial unit were used as additional devices supporting the integrated surveying. Another work [12] discusses precisely capturing geometrical changes in 2D using CRP. The proposed research method allows for high accuracy in determining displacements of the object's control points expressed even in sub-millimeter values. The presented method consists of a geometric system utilizing different camera positions and is mainly dedicated to indoor applications. The high accuracy was confirmed by a comparative study evaluating the displacements of the single-name points placed in a test stand using micrometers with a resolution of $0.01 \mathrm{~mm}$. The root-means-square (RMS) values of the differences between the predefined and evaluated displacements in the two directions (local X and Y) are 0.11 and $0.02 \mathrm{~mm}$, which closely predisposes this approach to metrological tasks. However, relatively small objects placed in a limited space were assessed in this case. A comparative study on the influence of control points number on CRP positioning is explained in [13]. Nevertheless, the studied solution concerns aerial photogrammetry using unmanned aerial vehicles (UAV). The obtained accuracies vary within a few centimeters, allowing the discussed solution to map the displacements of natural objects of considerable size (for example, open-pit mines, geotechnical structures, slopes, or embankments) [14]. All previously cited works together with [15] confirm the high usefulness of photogrammetric methods in industrial solutions, which require the exact determination of geometry elements of the examined objects. Recently, also low-cost solutions have become very popular in the precise dimensioning of things [16]. This approach uses inexpensive devices and technologies, competing with well-known, classic solutions provided by recognized manufacturers. The study [17] presented a low-cost CRP system for imaging building facades. Such a system uses a specially designed measuring set employing a camera with a laser distance meter making it possible to conduct measurements with millimeter-level accuracy. The authors used the self-developed software to assess the impact of various uncertainties (including camera calibration errors) on the surveying results. In this case, a crucial factor was recognizing the measurement set's geometrical parameters, which determines the absolute accuracy of the derived object model. CRP is also successfully used together with other techniques for remote detection of object geometry [18] - for instance, TLS. An example of such integration in archaeological applications is shown in [19]. The presented approach allows accurately mapping objects in 3D, treating precisely determined geometric data (distances between points) as a significant enhancement of a point cloud. A similar integration of data from remote sensing imaging and numerical terrain models is presented in [20]. In the accurate and reliable determination of the geometric parameters of engineering objects using photogrammetry, a crucial role plays not only the instrumental aspect but - perhaps above all - the methodological approach. Developing an optimal strategy for acquiring, processing, and analyzing measurement results is the pivotal problem of modern geomatics. The issues mentioned above motivated the authors to develop and test a CRP method based on adjusting the spatial control network located either on a test object or in its proximity. The article analyzes the accuracy of the proposed method, based on previous surveying such a linear control network using a measuring tape with a precision of $\pm 1 \mathrm{~mm}$.

\section{Materials and Methods}

The developed procedure is based on the classic stereo-pair of pictures taken for a test site using a popular camera. The control network consisting of linear values has been marked directly on the object, the points of which represent its characteristic elements. The control points gain Cartesian coordinates expressed in the local system encompassing the pictured object as well as the pixel coordinates determined for the left and right photograph. Then, the measured linear elements of the control network related to the local coordinates of selected points are adjusted by the least-squares method employing the Gauss-Markov model [21]. The adjusted positions of the control points are then used to set out the orientation and distortion parameters both for the left and right image, 
respectively. For the resulting center projections, the accuracy assessment is carried out considering the estimated errors in determining their parameters. As a result - the photogrammetrically derived linear elements of the control network are compared with the actual measures derived from the direct object survey.

To verify the developed method, we took control measurements in the university classroom, assuming as the test object classic an office desk equipped with some auxiliary elements. The control network oriented in the local XYZ coordinate encompassed all its characteristic points (object vertices). All linear components were measured three times with a surveying tape, thus determining their values with $\pm 1 \mathrm{~mm}$ precision. The $3 \mathrm{D}$ linear network established on the investigated object consisted of 12 points joined by 40 distances. Then, using a professional mirror-reflex camera, Canon EOS 500D [22], two photos were taken covering the item from its left and right side (Figures 1 and 2).

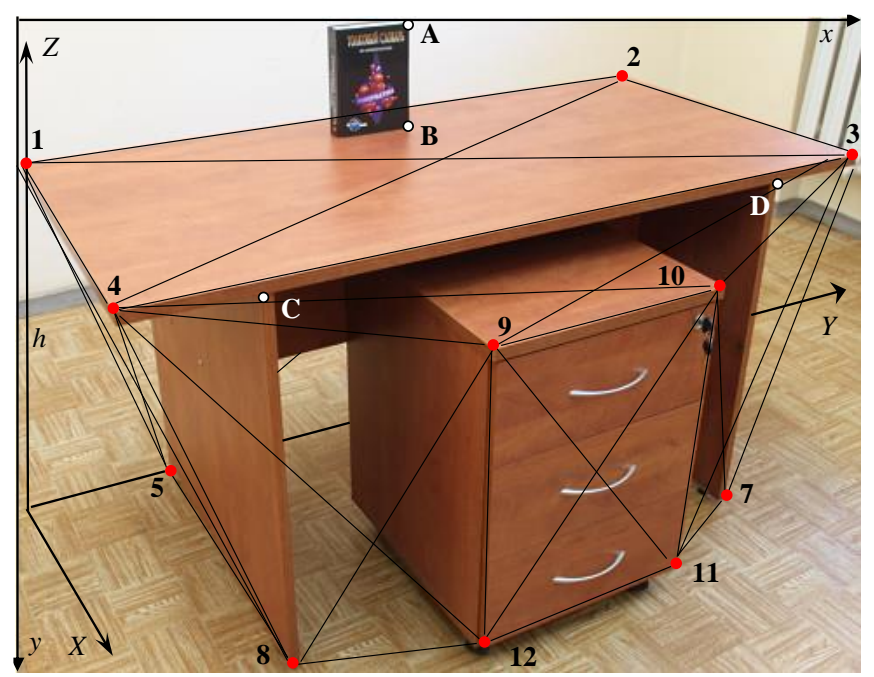

Figure 1. The left stereo picture with the $3 \mathrm{D}$ precise linear network on the object (the points 1..12, $A, B, C, D$ represent characteristic vertices of the object; $X, Y, Z$ define a local 3D coordinate system; $x, y$ represent pixel image coordinates, while $h$ is the object's approximate height)

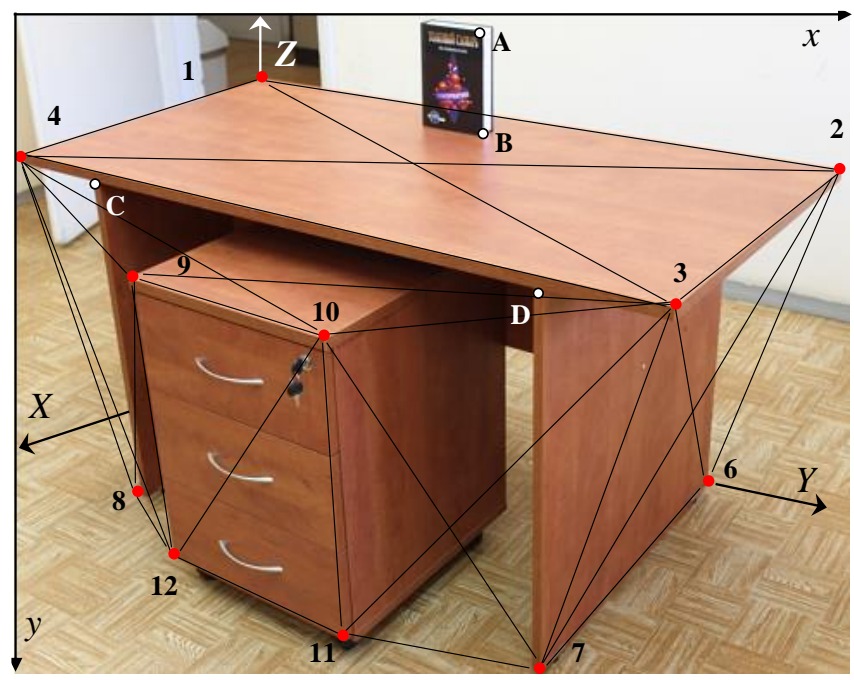

Figure 2. The right stereo picture with $3 \mathrm{D}$ precise linear network on the object (the points $1 . .12$ and $A, B, C, D$ represent characteristic vertices of the object; $\mathrm{X}, \mathrm{Y}, \mathrm{Z}$ define a local 3D coordinate system; $x, y$ represent pixel image coordinates). 
The key technical parameters of the camera used are presented in Table 1 [23].

Table 1. Key technical parameters of the camera Canon EOS 500D.

\begin{tabular}{|c|c|}
\hline Parameter & Description \\
\hline Type & $\begin{array}{l}\text { Digital AF/AE SLR camera with } \\
\text { built-in flash }\end{array}$ \\
\hline Recording media & $\begin{array}{l}\text { SD memory card, SDHC } \\
\text { memory card }\end{array}$ \\
\hline Image type & $\begin{array}{l}\text { JPEG, RAW (14-bit, Canon origi- } \\
\text { nal) }\end{array}$ \\
\hline Image sensor type & CMOS sensor \\
\hline Image sensor size & $22.3 \times 14.9 \mathrm{~mm}$ \\
\hline Resolution & $\begin{array}{l}\text { Effective pixels: approx. } 15.10 \\
\text { MPx } \\
\text { Total pixels: approx. } 15.50 \mathrm{MPx}\end{array}$ \\
\hline Color filter system & RGB primary color filter \\
\hline Low-pass filter & $\begin{array}{l}\text { Fixed position in front of the im- } \\
\text { age sensor }\end{array}$ \\
\hline Focus modes & $\begin{array}{l}\text { Servo mode; Auto-focus; Man- } \\
\text { ual focus }\end{array}$ \\
\hline Exposure control & $\begin{array}{l}\text { Full Auto, Portrait, Landscape, } \\
\text { Close-up, Sports, Night Portrait, } \\
\text { Flash Off, } \\
\text { Creative Auto, Program), shut- } \\
\text { ter-priority AE, aperture-prior- } \\
\text { ity AE, auto depth-of-field AE, } \\
\text { manual exposure, E-TTL II auto- } \\
\text { flash, movie shooting }\end{array}$ \\
\hline
\end{tabular}

It should be added that the camera is commonly available on the market, emphasizing is the low-cost feature of the presented method. For the experiment, the photos were taken using the classic indoor shooting program at short distances to the subject (portrait function at a fixed focal length, f/2 aperture, shutter speed 1: $200 \mathrm{~s}$, ISO 400, no flash).

\section{Results}

As it is shown in figures 1 and 2, the reference frame $X, Y, Z$ is connected with the network, assuming the $Z$-axis passes through point 1 perpendicularly to the plane with points $1,2,4$. The axis $Y$ is parallel to the section $|1-2|$, and the third orthogonal axis $X$ is parallel to $|1-4|$. Hence, the three connecting reference sections with network points 1,2 and 4 obtained coordinates: $1(0,0, h), 2(0,|1-2|, h), 4(|1-4|, 0, h)$. The sections $|1-2|$ and $|1-4|$ represent measured distances between points 1 and 2 as well as 1 and 4 , respectively. The value $\mathrm{h}$ is the approximate height of the object. The approximate coordinates $\mathrm{X}, \mathrm{Z}, \mathrm{Y}$ of all remaining points $3,5, \ldots, 12$ are computed by solving the network consisting of 40 measured distances between all points. 


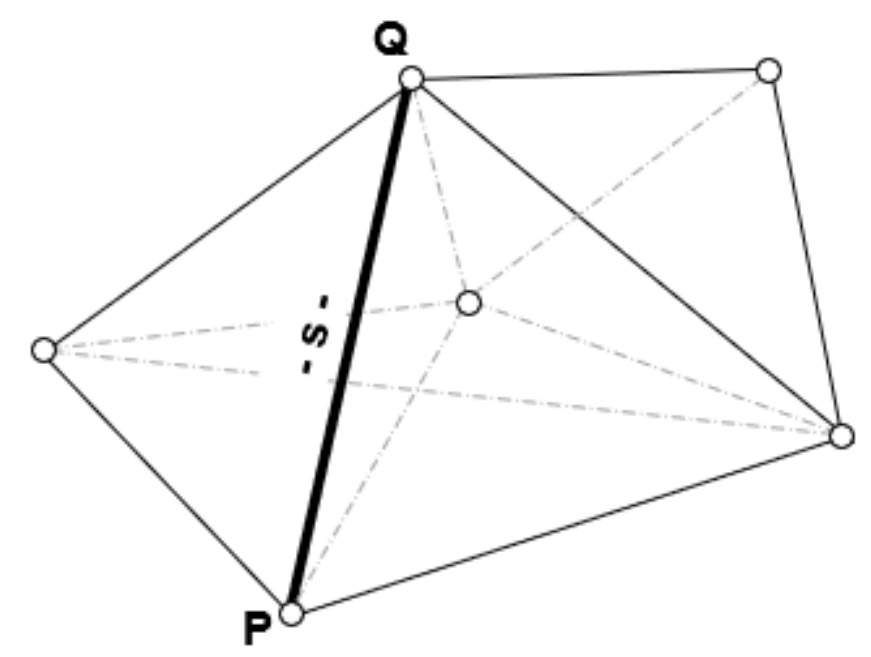

Figure 3. Exemplary spatial network.

To evaluate the proposed approach, we consider an example 3D network presented in Figure 3, which exemplifies a standard spatial network used in land surveying. The observational linear equation of the measured spatial distance $s$ between any two spatial network points $\mathrm{P}\left(\mathrm{X}_{\mathrm{P}}, \mathrm{X}_{\mathrm{P}}, \mathrm{X}_{\mathrm{P}}\right)$ and $\mathrm{Q}\left(\mathrm{X}_{\mathrm{Q}}, \mathrm{X}_{\mathrm{Q}}, \mathrm{X}_{\mathrm{Q}}\right)$ is based on the well-known formula used in three-dimensional surveying [24]:

$$
\begin{aligned}
v= & -\frac{X_{Q}-X_{P}}{s_{0}} d X_{P}-\frac{Y_{Q}-Y_{P}}{s_{0}} d Y_{P}-\frac{Z_{Q}-Z_{P}}{s_{0}} d Z_{P} \\
& +\frac{X_{Q}-X_{P}}{s_{0}} d X_{Q}+\frac{Y_{Q}-Y_{P}}{s_{0}} d Y_{Q}+\frac{Z_{Q}-Z_{P}}{s_{0}} d Z_{Q}-\left(s-s_{0}\right), \quad \sigma_{s}
\end{aligned}
$$

where: $d X_{P}, d Y_{P}, d Z_{P}$, and $d X_{Q}, d Y_{Q}, d Z_{Q}$ are corrections of the approximate coordinates $X_{P}$, $\mathrm{Y}_{\mathrm{P}}, \mathrm{Z}_{\mathrm{P}}$ and $\mathrm{X}_{\mathrm{Q}}, \mathrm{Y}_{\mathrm{Q}}, \mathrm{Z}_{\mathrm{Q}}$ of the points $P$ and $Q$, respectively, $\mathrm{v}$ is the random error of distance measurement $s$ with zero value expectation and known standard deviation, equals $\sigma_{s}=1$ $\mathrm{mm}$, so is an approximate value of the measured distance s:

$$
s_{0}=\sqrt{\left(X_{Q}-X_{P}\right)^{2}+\left(Y_{Q}-Y_{P}\right)^{2}+\left(Z_{Q}-Z_{P}\right)^{2}}
$$

The Gauss-Markov observational model [25-26] for all measured spatial distance observations $\mathrm{s}_{1}, \mathrm{~s}_{2}, \ldots, \mathrm{Sn}, n=40$, (Fig. 1, Fig 2) is completed regarding the equation (1):

$$
\mathbf{v}=\mathbf{A x}-\left(\mathbf{s}-\mathbf{s}_{0}\right), \mathbf{\Sigma}_{\mathbf{s}}
$$

where: $\mathrm{x}$ - vector of unknown corrections: $d Y_{2}, d X_{3}, d Y_{3}, d Z_{3}, d X_{4}, d Y_{4}, d X_{5}, d Y_{5}, d Z_{5}, \ldots$, $d X_{12}, d Y_{12}, d Z_{12}, v$ - vector of unknown observational error residuals $\mathrm{v} 1_{1}, \mathrm{v}_{2}, \ldots, \mathrm{vn}_{\mathrm{n}}, A$ - known design matrix, $s$ - vector of observations $\mathrm{s}_{1}, \mathrm{~s}_{2}, \ldots, \mathrm{s}_{n}, \mathrm{~s}_{0}$ - vector of approximate values of observations $\mathrm{s}_{0}, 1, \mathrm{~s} 0,2, \ldots, \mathrm{s} 0, \mathrm{n}, \sigma_{\mathrm{s}}$ - diagonal covariance matrix of observations $\mathrm{s}$, composed from standard deviations $\sigma_{\mathrm{s}, 1}, \sigma_{\mathrm{s}, 2}, \ldots, \sigma_{\mathrm{s}, \mathrm{n}}$.

In the adjustment, the network is connected with the reference frame by three coordinates $X_{1}, Y_{1}, Z_{1}$ of point 1 , two coordinates $X_{2}, Z_{2}$ of point 2 , and one coordinate $Z_{4}$ of point 4 . These coordinates are constant, it means $d X_{1}=d Y_{1}=d Z_{1}=0, d X_{2}=d Z_{2}=0$, $d Z_{4}=0$. 
The least-squares solution of the Gauss-Markov model (8): $v^{T} \sum_{s}^{-1} v=\min$ is given by well-known formulas [27]:

$$
\mathbf{x}=\boldsymbol{\Sigma}_{\mathbf{x}} \mathbf{A}^{T} \mathbf{\Sigma}_{\mathbf{s}}^{-1}\left(\mathbf{s}-\mathbf{s}_{0}\right)
$$

where the equations:

$$
\begin{aligned}
& \boldsymbol{\Sigma}_{\mathbf{x}}=\left(\mathbf{A}^{T} \boldsymbol{\Sigma}_{\mathbf{s}}^{-1} \mathbf{A}\right)^{-1} \\
& \boldsymbol{\Sigma}_{\mathbf{v}}=\boldsymbol{\Sigma}_{\mathbf{s}}-\mathbf{A} \boldsymbol{\Sigma}_{x} \mathbf{A}^{T}
\end{aligned}
$$

represent the covariance matrices of $x$ and $v$, respectively.

The maximal value of the standard deviations $\sigma x, \sigma Y, \sigma Z$ of the adjusted coordinates $Y_{2}+d Y_{2}, X_{3}+d X_{3} Y_{3}+d Y_{3}, Z_{3}+d Z_{3}, X_{4}+d X_{4}, Y_{4}+d Y_{4}, X_{5}+d X_{5}, Y_{5}+d Y_{5}, Z_{5}+d Z_{5} \ldots X_{12}+d X_{12}, Y_{12}+d Y_{12}$, $Z_{12}+Z_{12}$, taken from diagonal elements of the covariance matrix (5) is equal $1.2 \mathrm{~mm}$.

The adjusted positions of the network point $1,2, \ldots 12$ are used as so-called control points for the determination of the orientation and distortion parameters of the left and right stereo photos in the object reference frame $(X, Y, Z)$.

\subsection{Accuracy of the photogrammetric intersection}

\subsubsection{The photogrammetric central projection}

The photogrammetric central projection is a projection of the three-dimensional object space $(X, Y, Z)$ onto a two-dimensional image plane ( $x, y)$ (Fig.1, Fig.2). The central projection equations $(X, Y, Z) \rightarrow(x, y)$ are given by following [28-30]:

$$
\begin{aligned}
x=x_{0}-f_{s} & \frac{r_{11}\left(X-X_{0}\right)+r_{12}\left(Y-Y_{0}\right)+r_{13}\left(Z-Z_{0}\right)}{r_{31}\left(X-X_{0}\right)+r_{32}\left(Y-Y_{0}\right)+r_{33}\left(Z-Z_{0}\right)} \\
& -\left[k_{1}\left(\left(x-x_{0}\right)^{2}+\left(y-y_{0}\right)^{2}\right)+k_{2}\left(\left(x-x_{0}\right)^{2}+\left(y-y_{0}\right)^{2}\right)^{2}\right]\left(x-x_{0}\right) \\
& -k_{3}\left[3\left(x-x_{0}\right)^{2}+\left(y-y_{0}\right)^{2}\right]-2 k_{4}\left(x-x_{0}\right)\left(y-y_{0}\right) \\
y=y_{0}+f_{s} & \frac{r_{21}\left(X-X_{0}\right)+r_{22}\left(Y-Y_{0}\right)+r_{23}\left(Z-Z_{0}\right)}{r_{31}\left(X-X_{0}\right)+r_{32}\left(Y-Y_{0}\right)+r_{33}\left(Z-Z_{0}\right)} \\
& -\left[k_{1}\left(\left(x-x_{0}\right)^{2}+\left(y-y_{0}\right)^{2}\right)+k_{2}\left(\left(x-x_{0}\right)^{2}+\left(y-y_{0}\right)^{2}\right)^{2}\right]\left(y-y_{0}\right) \\
& -2 k_{3}\left(x-x_{0}\right)\left(y-y_{0}\right)-k_{4}\left[\left(x-x_{0}\right)^{2}+3\left(y-y_{0}\right)^{2}\right]
\end{aligned}
$$

where $X, Y, Z$ are coordinates of an object point $P ; x, y$ are pixel image coordinates of the projected point $P ; Y_{0}, Y_{0}, Z_{0}$ are coordinates of the central projection point $\mathrm{O}_{;} \mathrm{x}_{0}, \mathrm{y}_{0}$ are image pixel coordinates of the central point $\mathrm{O} ; k_{1}, k_{2}, k_{3}, k_{4}$, are parameters of the camera distortion, $\mathrm{fs}=\mathrm{f} / \mathrm{s}, \mathrm{f}$ is the focal length of the camera, $\mathrm{s}$ is the CCD cell size of the camera, and:

$$
\left[\begin{array}{lll}
r_{11} & r_{12} & r_{13} \\
r_{21} & r_{22} & r_{23} \\
r_{31} & r_{32} & r_{33}
\end{array}\right]=\left[\begin{array}{ccc}
\cos \phi \cos \kappa & \cos \omega \sin \kappa+\sin \omega \sin \phi \cos \kappa & \sin \omega \sin \kappa-\cos \omega \sin \phi \cos \kappa \\
-\cos \phi \sin \kappa & \cos \omega \cos \kappa-\sin \omega \sin \phi \sin \kappa & \sin \omega \cos \kappa+\cos \omega \sin \phi \sin \kappa \\
\sin \phi & -\sin \omega \cos \phi & \cos \omega \cos \phi
\end{array}\right]
$$

is the matrix of rotation $(\omega, \varphi, \chi)$ of the object reference frame $(X, Y, Z)$ related to the camera reference frame $\left(Y_{k}, Y_{k}, Z_{k}\right)$. The frame's origin $\left(Y_{k}, Y_{k}, Z_{k}\right)$ is the central projection point 
$O$, the axes $Y_{k}, Y_{k}$ run parallel to the sections of the CCD frame, and $Z_{k}$ is perpendicular to the CCD frame. The six parameters $Y_{0}, Y_{0}, Z_{0}, \omega, \varphi, \varkappa$ define the external orientation of the camera reference frame $\left(Y_{k}, Y_{k}, Z_{k}\right)$ concerning the object reference frame $(X, Z, Y)$. The three parameters $x_{0}, y_{0}, f=f_{s}$ define the internal orientation of the image referring the camera reference frame $\left(Y_{k}, Y_{k}, Z_{k}\right)$.

\subsection{The photogrammetric spatial resection}

The photogrammetric spatial resection method allows for determining orientation ( $\mathrm{Y}_{0}, \mathrm{Y}_{0}$, $\left.\mathrm{Z}_{0}, \omega, \varphi, \varkappa, \mathrm{x}_{0}, \mathrm{y}_{0}, \mathrm{f}_{\mathrm{s}}\right)$ and distortion $\left(\mathrm{k}_{1}, \mathrm{k}_{2}, \mathrm{k}_{3}, \mathrm{k}_{4}\right)$ parameters of an image based on the known control points deployed on the object [31]. In the case of the left image, the 13 orientation and distortion parameters were computed solving the set of 22 equations (7), (8) for eleven visible control points $1,2, \ldots, 12$ (Fig.1) by the least-squares method. The camera's determined position $\left(\mathrm{Y}_{0}, \mathrm{Y}_{0}, \mathrm{Z}_{0}\right)$ in the left $(\mathrm{L})$ is shown in Fig. 3. The pixel coordinates $\mathrm{X}_{\text {comp, }} \mathrm{ycomp}$ of the control points computed after adjustment according to the equations (7), (8) are not exactly equal to the measured values $x, y$. The residual mean squares (RMS) of the obtained pixel coordinates residuals $\varepsilon_{\mathrm{x}}=\mathrm{x}-\mathrm{x}$ comp, $\varepsilon_{\mathrm{y}}=\mathrm{y}-\mathrm{ycomp}$ are equal to 1.8 and 2.0 in pixels, respectively.

In the case of the right image, the 13 orientation and distortion parameters were computed solving a set of 22 equations (7), (8) for eleven visible control points 1,2,..,12 (Fig.2) by the least-squares method. The camera's determined position ( $Y 0, Y 0, Z 0)$ in the right (R) is shown in Fig. 4. The RMS for obtained pixel coordinates residuals $\varepsilon_{x}=x-x_{c o m p}, \varepsilon_{y}=y-$ ycomp are equal 1.3 and 1.6 in pixel values, respectively.

Due to the nonlinear least-squares problem, the approximate values of the parameters were computed using the Direct Linear Transformation (DLT) method [32].

a) view fom above

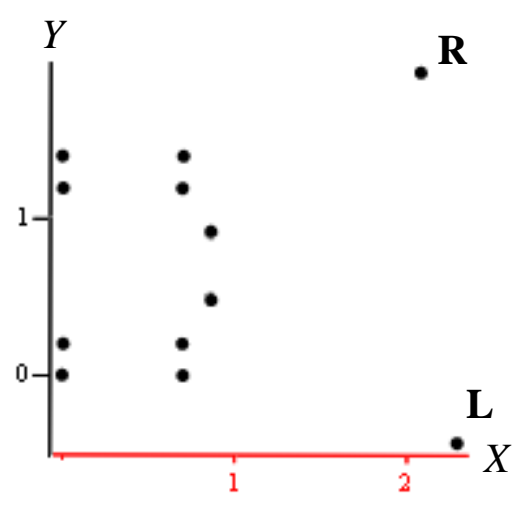

b) perspective view

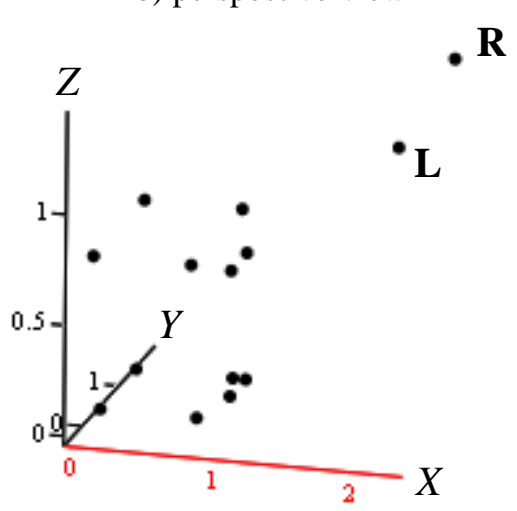

Figure 4. Determined camera locations L-left and R-right with 12 control points deployed on the test object.

\subsection{The photogrammetric triangulation}

The photogrammetric triangulation is a method of simultaneous determination of the orientation and distortion parameters of the stereo or multi-images as well as the coordinates X, Y, Z of new so-called tie points located on the object [33]. In our experiment, we chose only one tie point $A$ (Fig. 1 and 2). In this case, the 26 orientation and distortion parameters of the left and right stereo images plus three coordinates $X_{A}, Y_{A}, Z_{A}$ of the tie point $\mathrm{A}$ were computed solving the set of 48 equations (7) and (8) by the least-squares 
method [34]: 24 equations for twelve visible points on the left image 1,2,.,12, A and 24 equations for twelve visible points on the right image $1,2, \ldots, 12, A$. The RMS values for obtained pixel coordinates residuals $\varepsilon_{x}, \varepsilon_{y}$ are equal to 1.5 and 2.1 in pixel values, respectively. The determined positions of the camera in the left and right position concerning the 12 network points shown in Fig. 3.

\subsection{The accuracy of the photogrammetric intersection}

The photogrammetric intersection is a method of computing the coordinates of object points based on the spatially oriented stereo or multi-images referenced in the object reference system $X, Y, Z$ [34]. For example, the coordinates $X_{\mathrm{B}}, Y_{\mathrm{B}}, Z_{\mathrm{B}}$ of the new object point $B$ (Fig. 1, Fig. 2) are computed solving four equations (7), (8) by least squares method: two equations for measured pixel coordinates of the projected point $B$ on the left image (Fig.1) and two equations for projected point $B$ on the right image (Fig.2). The computed spatial distance between points $A\left(X_{A}, Y_{A}, Z_{A}\right)$ and $B\left(X_{B}, Y_{B}, Z_{B}\right)$ is equal to $\left[\left(X_{B}-X_{A}\right)^{2}+\left(Y_{B}\right.\right.$ $\left.\left.-Y_{A}\right)^{2}+\left(Z_{B}-Z_{A}\right)^{2}\right]^{1 / 2}=0.210 \mathrm{~m}$. Distance between these points measured by tape is also equal to $0.210 \mathrm{~m}$. So, in this case, the error of the photogrammetrically measured distance between two points $\mathrm{A}$ and $\mathrm{B}$ on the object is equal to zero.

In the second example, the distance between points $C$ and $D$ is computed (Fig. 1 and 2 ). The coordinates of points $C$ and $D$ were computed in the same way as those of point $\mathrm{B}$. The computed spatial distance between photogrammetrically determined points $\mathrm{C}$ (xC, $\left.\mathrm{yC}_{\mathrm{C}} \mathrm{zC}\right)$ and $\mathrm{D}\left(\mathrm{x}_{\mathrm{D}}, \mathrm{yD}_{\mathrm{D}} \mathrm{zD}\right)$ is equal $\left[\left(\mathrm{X}_{\mathrm{D}}-\mathrm{X}_{\mathrm{C}}\right) 2+\left(\mathrm{Y}_{\mathrm{D}}-\mathrm{Y}_{\mathrm{C}}\right) 2+\left(\mathrm{Z}_{\mathrm{D}}-\mathrm{Z}_{\mathrm{C}}\right)^{2}\right]^{1 / 2}=0.989 \mathrm{~m}$. Distance between these points measured by tape is equal to $0.990 \mathrm{~m}$. In this experiment error of the photogrammetrically measured distance between two points $C$ and $D$ on the object is equal to $1 \mathrm{~mm}$.

\section{Discussion}

Close-range photogrammetry is used in many engineering fields. Employing it for the dimensioning of objects and assessing displacement values requires developing and testing an appropriate strategy. In the case of small objects, more precise control points can be set out by founding and adjusting a three-dimensional linear network on the object. Our experiment shows that the adjusted positions of the control points are determined with high accuracy of about $\pm 1 \mathrm{~mm}$. The photogrammetric $3 \mathrm{D}$ model derived regarding the control points deployed directly on the examined structure and the two images taken with the non-metric camera Canon EOS 500D demonstrates high - one-millimeter accuracy. The distance between two points selected on the spatial model directly equals realobject lengths. Hence, the elaborated methodology allows for dimensioning engineering objects in tasks where high accuracies are needed.

What is more, the proposed approach is progressive and can be freely modified to be combined with other surveying technologies. For example, it can be utilized while dimensioning indoor spaces for the area and volume calculations. Another possibility is to use such technology in displacement monitoring, control measurements, or finishing works on a construction site.

In contemporary geomatics, one of the deciding factors while choosing a particular surveying method is its availability and time consumption. In the discussed case, both conditions are fulfilled. Using a non-metric, commonly used camera makes the solution low-cost. Also, data processing is not time-consuming, and the algorithm can be run on a standard computer. The application is possible for each object, where a threedimensional linear network can be established. Such a point deployment can be possible in different ways - either by selecting characteristic object points (for example, its vertices and edges) or by projecting light spots using a common laser emitter. The network can be measured 
using a tape or hand-held laser distance meter as well as - in case of poorer object availability - with a total station offering a reflectorless distance surveying option.

The limitation in using the developed method may be poor lighting conditions on the site, insufficient resolution of the camera, and the inability to cover the object with a control network. It can be mentioned that the location of network points may refer to both characteristic ends of the measured object as well as points marked using available laser projectors. These items are the subject of further research conducted by the authors.

Author Contributions: Conceptualization E.O., methodology K.K. and E.O., validation K.K. and E.O., writing-original draft preparation K.K. All authors have read and agreed to the published version of the manuscript.

Funding: The authors declare no funding.

Data Availability Statement: The data presented in this study is available on request from the co-author Prof. Edward Osada: edward.osada@dsw.edu.pl.

Conflicts of Interest: The authors declare no conflict of interest.

\section{References}

[1] Pozzoli A., Mussio L., Quick solutions particularly in close range photogrammetry, The International Archives of the Photogrammetry, Remote Sensing and Spatial Information Sciences, Vol. XXXIV, Part 5/W12, 2012, pp. 273-278.

[2] Osada E., Sośnica K., Borkowski A., Owczarek-Wesołowska M., Gromczak A., Direct Georeferencing Method for Terrestrial Laser Scanning Using GNSS Data and the Vertical Deflection from Global Earth Gravity Models, Sensors, 2017, vol. 17, 1489.

[3] Remondino F., El-Hakim S., Image-Based 3D Modelling: A Review, The photogrammetric Record, 2006, vol. 21, pp. $269-291$.

[4] Luhmann T., Robson S., Kyle S., Boehm J., Close range Photogrammetry and 3D Imaging, second ed. Walter de Gruyter GmbH, Berlin/Boston, 2013, pp. 221-253.

[5] Cyganek B., Siebert J.P., An introduction to 3D Computer Vision, Techniques and Algorithms, John Wiley \& Sons Ltd., 2009, pp. 323-342.

[6] Mataa E., Hernandeza M.A., Cardenala J., Pereza J.L., Assisted control point measurement for close range photogrammetry, International Archives of Photogrammetry, Remote Sensing and Spatial Information Sciences, 2012, vol. XXXIX-B5, pp. 369-373.

[7] Osada E., Owczarek-Wesołowska M.,. Ficner M, Kurpinski G., TotalStation/GNSS/EGM integrated geocentric positioning method, Survey Review, 2017, vol. 49, pp. 206-211.

[8] Luhmann T., Robson S., Kyle S., Harley I., Close Range Photogrammetry - principles, techniques and applications, Whittles Publishing, Dunbeath, Scotland, UK, 2011, pp. 135-181.

[9] Revilla-León M., Att W., Özcan M., Rubenstein J., Comparison of conventional, photogrammetry, and intraoral scanning accuracy of complete-arch implant impression procedures evaluated with a coordinate measuring machine, The Journal of Prosthetic Dentistry, 2021, vol. 125, Issue 3, pp. 470-478.

[10] Sapirstein P., A high-precision photogrammetric recording system for small artifacts, Journal of Cultural Heritage, 2018, vol. 31, pp. 33-45.

[11] Yakar M., Yilmaz H.M., Mutluoglu O., Close range photogrammetry and robotic total station in volume calculation, International Journal of the Physical Sciences, 2010, vol. 5. 2, pp. 086-096.

[12] Navarro S., Lerma J.L., Accuracy analysis of a mobile mapping system for close range photogrammetric projects, measurement, 2016, vol. 93, pp.148-156.

[13] M. Khalil A., Two-dimensional displacement measurement using static close range photogrammetry and a single fixed camera, Alexandria Engineering Journal, 2011, vol. 50, pp. 219-227.

[14] Ding-bang Z., Yi Z., Tao Ch., Yuan M., Kun F., Ankit G., Akhil G., Measurement of displacement for open pit to underground mining transition using digital photogrammetry, Measurement, 2017, vol. 109, pp. 187-199.

[15] YeN., Zhu H., Wei M., Zhang L., Accurate and dense point cloud generation for industrial measurement via target-free photogrammetry, Optics and Lasers in Engineering, 2021, vol. 140, https://doi.org/10.1016/j.optlaseng.2020.106521 (access: 17.02.2022).

[16] Agüera-Vega F., Carvajal-Ramírez F., Martínez-Carricondo P., Assessment of photogrammetric map-ping accuracy based on variation ground control points number using unmanned aerial vehicle, Measurement, 2017, vol. 98, pp. $221-227$.

[17] Ćmielewski K., Karsznia K., Kuchmister J., Gołuch P., Wilczyńska I., Accuracy and functional assessment of an original low-cost fibre-based inclinometer designed for structural monitoring, Open Geosciences, 2020, vol. 12, issue 1, pp. 1052-1059.

[18] Esmaeili F., Varshosaz M., Ebadi H., Displacement measurement of the soil nail walls by using close range photogrammetry and introduction of CPDA method, Measurement, 2013, vol. 46, pp. 3449-3459. 
[19] Ordóñez C., Martínez J., Arias P., Armesto J., Measuring building façades with a low-cost close-range photogrammetry system, Automation in Construction, 2010, vol. 19, pp. 742-749.

[20] Lerma J.L., Navarro S., Cabrelles M., Villaverde V., Terrestrial laser scanning and close range photogrammetry for 3D archaeological documentation: the Upper Palaeolithic Cave of Parpallo' as a case study, Journal of Archaeological Science, 2010, vol. 37, pp. 499-507.

[21] Christensen R. In: R. Christensen (Ed.), General Gauss-Markov models. Plane Answers to Complex Questions: The Theory of Linear Models. 4th ed., New York: Springer, 2011, pp. 237-266.

[22] Canon EOS 500D Specifications, Technical Brochure, Canon Hongkong Company Limited, 2008.

[23] Esmaeili F., Varshosaz M., Ebadi H., Displacement measurement of the soil nail walls by using close range photogrammetry and introduction of CPDA method, Measurement, 2013, vol. 46, pp. 3449-3459.

[24] Forstner W., Wrobel B.P., Photogrammetric Computer Vision, Statistics, Geometry, Orientation and Reconstruction, Springer International Publishing Switerzland, 2016, pp. 195-246.

[25] Isotalo J, Linear estimation and prediction in the general Gauss-Markov model, Academic dissertation, University of Tampere, Finland, 2007, https://trepo.tuni.fi/bitstream/handle/10024/67741/978-951-44-7018-9.pdf?sequence=1 (access: 24.02.2022).

[26] Christensen R. General Gauss-Markov models. In: R. Christensen (Ed.), Plane Answers to Complex Questions: The Theory of Linear Models. 4th ed., New York: Springer, 2011, pp. 237-266.

[27] R. Jiang, D.V. Jauregui, K.R. White, Close-range photogrammetry applications in bridge measurement: Literature review, Measurement, 2008, vol. 41, pp. 823-834.

[28] Luhmann T., Close range photogrammetry for industrial applications, ISPRS Journal of Photogrammetry and Remote Sensing, 2010, vol.65, pp. 558-569.

[29] Jiang R., Jáuregui D.V., White K.R., Close-range photogrammetry applications in bridge measurement: Literature review, measurement, vol. 41, issue 8, 2008, pp. 823-834.

[30] Luhmann T., Robson S., Kyle S., Harley I., Close Range Photogrammetry Principles, techniques and applications, Whittles Publishing, Dunbeath, Scotland, UK, 2011, pp. 135-181.

[31] Wang X, Clarke T.A. Separate adjustment of close-range photogrammetric measurements. ISPRS, 1998, vol. XXXII, part 5, pp. 177-184.

[32] El-Ashmawy K. L. A., Using direct linear transformation (DLT) method for aerial photogrammetry applications, Geodesy and Cartography, 2018, vol. 44(3), pp. 71-79.

[33] Schenk K., From point-based to feature-based aerial triangulation, ISPRS Journal of Photogrammetry E Remote Sensing, 2004, vol. 58, pp. $315-329$.

[34] Bellamy C., Watterson G., Least Squares Adjustment of networks, Survey Review, 1970, vol. 20:156, pp. 250-258. 